\title{
Severe Hepatic Sinusoidal Obstruction Syndrome in a Child Receiving Vincristine, Actinomycin-D, and Cyclophosphamide for Rhabdomyosarcoma: Successful Treatment with Defibrotide
}

\author{
Aery Choi, MD \\ Young Kyung Kang, MD \\ Sewon Lim, MD \\ Dong Ho Kim, MD, MS \\ Jung Sub Lim, MD, PhD \\ Jun Ah Lee, MD, PhD
}

Department of Pediatrics,

Korea Cancer Center Hospital, Seoul, Korea
Hepatic sinusoidal obstruction syndrome (SOS) is a life-threatening syndrome that generally occurs as a complication after hematopoietic stem cell transplantation or, less commonly, after conventional chemotherapy. Regarding SOS in rhabdomyosarcoma patients who received conventional chemotherapy, the doses of chemotherapeutic agents are associated with the development of SOS. Several cases of SOS in rhabdomyosarcoma patients after receiving chemotherapy with escalated doses of cyclophosphamide have been reported. Here, we report on a 9-year-old female with rhabdomyosarcoma who developed severe SOS after receiving chemotherapy consisting of vincristine, actinomycin-D, and a moderate dose of cyclophosphamide. She was treated successfully with defibrotide without sequelae to the liver.

Correspondence: Jun Ah Lee, MD, PhD

Department of Pediatrics,

Korea Cancer Center Hospital, 75 Nowon-ro,

Nowon-gu, Seoul 01812, Korea

Tel: 82-2-970-1248

Fax: 82-2-970-1970

E-mail: junahlee@kcch.re.kr

Received March 3, 2016

Accepted March 22, 2016

Published Online March 30, 2016

\section{Key words}

Hepatic veno-occlusive disease,

Rhabdomyosarcoma, Defibrotide

\section{Introduction}

Hepatic sinusoidal obstruction syndrome (SOS), formerly known as veno-occlusive disease, is a life-threatening syndrome [1-3]. SOS generally occurs as a complication after high-dose chemotherapy associated with hematopoietic stem cell transplantation (HSCT) [2]. Development of fatal SOS related to conventional chemotherapy is rare. While SOS in patients undergoing HSCT is life-threatening, cases in patients receiving conventional chemotherapy are mild and recover spontaneously. We report on a case of a severe hepatic SOS in a patient on chemotherapy consisting of vincristine, actinomycin-D, and a moderate dose of cyclophosphamide for rhabdomyosarcoma. 


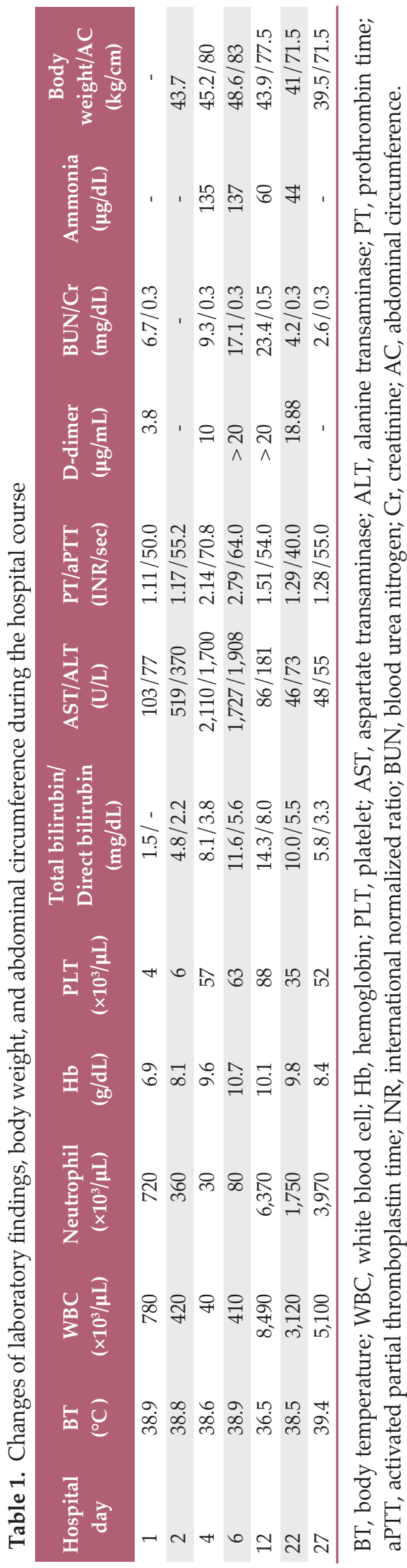

\section{Case Report}

A 9-year-old female developed fever, jaundice, and right upper quadrant abdominal pain. Two months earlier, she was diagnosed with rhabdomyosarcoma of the right deltoid and triceps muscles and received two courses of VAC chemotherapy (vincristine, $1.5 \mathrm{mg} / \mathrm{m}^{2} /$ day; actinomycin-D, $1.35 \mathrm{mg} / \mathrm{m}^{2} /$ day; cyclophosphamide, $1.2 \mathrm{~g} / \mathrm{m}^{2} /$ day for 3 days) every 3 weeks. She underwent wide excision of the tumor and proceeded with postoperative VAC chemotherapy. On the eighth day of the fourth VAC chemotherapy, she complained of mild abdominal pain and developed a neutropenic fever (white blood cell [WBC], $0.78 \times 10^{3} / \mu \mathrm{L}$; hemoglobin $[\mathrm{Hb}], 6.9 \mathrm{~g} / \mathrm{dL}$; platelets, $4 \times 10^{3} / \mu \mathrm{L}$ ) and was immediately started on intravenous broad-spectrum antibiotics (piperacillin and tazobactam), transfusion (red blood cells, platelets), and granulocyte colony stimulating factor. Her blood tests showed mild elevation of liver enzymes (aspartate transaminase [AST], $103 \mathrm{U} / \mathrm{L}$; alanine transaminase [ALT], $77 \mathrm{U} / \mathrm{L})$ and total bilirubin $(1.5 \mathrm{mg} / \mathrm{dL})$. The following day, her abdominal pain was aggravated with persistent fever, along with laboratory findings of pancytopenia (WBC, $0.42 \times 10^{3} / \mu \mathrm{L} ; \mathrm{Hb}, 8.1 \mathrm{~g} / \mathrm{dL}$; platelet, $\left.6 \times 10^{3} / \mu \mathrm{L}\right)$, a marked increase in liver enzymes (AST, $519 \mathrm{U} / \mathrm{L} ; \mathrm{ALT}, 370 \mathrm{U} / \mathrm{L}$ ), hyperbilirubinemia (total bilirubin, $4.8 \mathrm{mg} / \mathrm{dL}$ ), and prolonged prothrombin time (1.17 international normalized ratio) and active partial thromboplastin time (55.2 seconds). At this stage, her physical examination showed hepatomegaly, $3.5 \mathrm{~cm}$ distal from the right costal margin. Under the impression of sepsis, antibiotics were changed to vancomycin and meropenem with addition of intravenous immunoglobulin. However, her fever peaked to $38.9^{\circ} \mathrm{C}$ on hospital day (HD) 3 , and she developed severe abdominal pain.

Physical examination detected icteric sclerae and aggravated hepatomegaly with abdominal distension, and her body weight was increased by $6.1 \%(2.5 \mathrm{~kg})$. Laboratory results were abnormal: AST/ALT 1,172/810 U/L, total/ direct bilirubin $6.1 / 3.1 \mathrm{mg} / \mathrm{dL}$ (Table 1). Abdominal Doppler ultrasonography (US) showed reversed blood flow of the right portal vein (Fig. 1A). Based on the SOS diagnosis, treatment was initiated with defibrotide $(32 \mathrm{mg} / \mathrm{kg} /$ day in four divided doses). She was transferred to the intensive care unit, and her condition became unstable with chest radiography showing diffuse pleural effusion. She became irritable and did not recognize her mother and medical personnel. Her liver enzyme levels peaked to an AST of 2,110 U/L and ALT of 1,908 U/L, and her total bilirubin and direct bilirubin were elevated up to $15.1 \mathrm{mg} / \mathrm{dL}$ and $8.6 \mathrm{mg} / \mathrm{dL}$, respectively. Her ammonia level was also increased $(135 \mu \mathrm{g} / \mathrm{dL})$. She was diagnosed with hepatic encephalopathy with addition of 

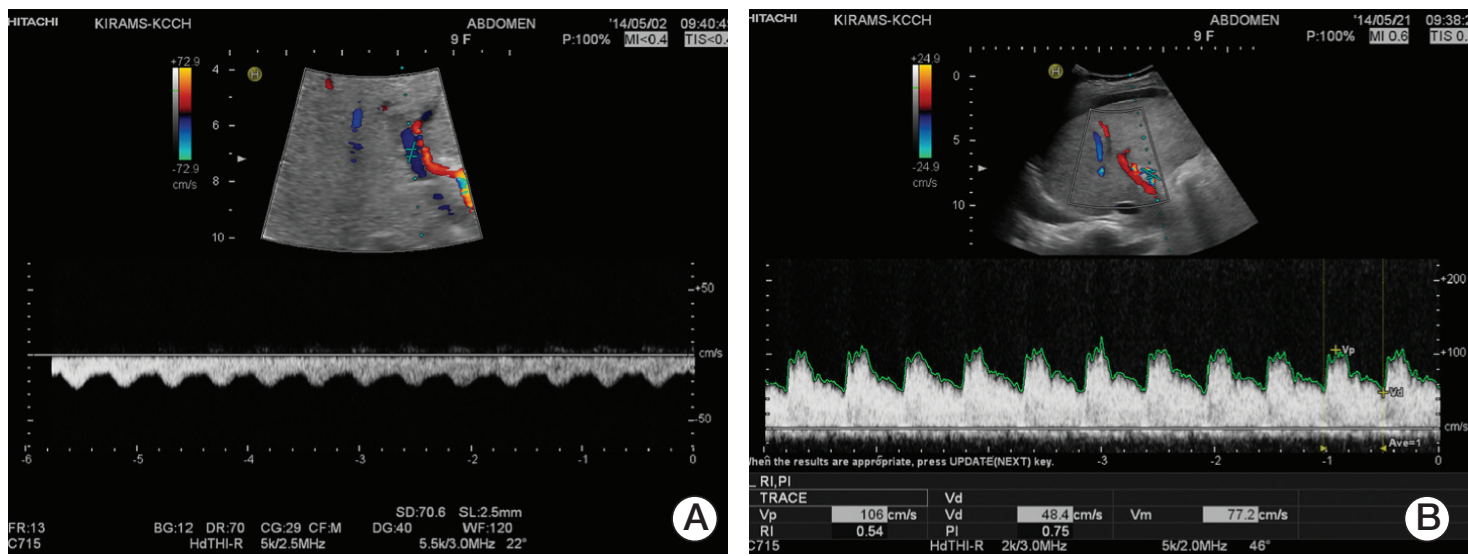

Fig. 1. Abdominal Doppler ultrasonography performed on hospital day (HD) 3 (A) and HD 22 (B). Blood flow of the right portal vein was reversed (A) upon sinusoidal obstruction syndrome diagnosis. Direction of blood flow was normalized (B) after treatment with defibrotide.

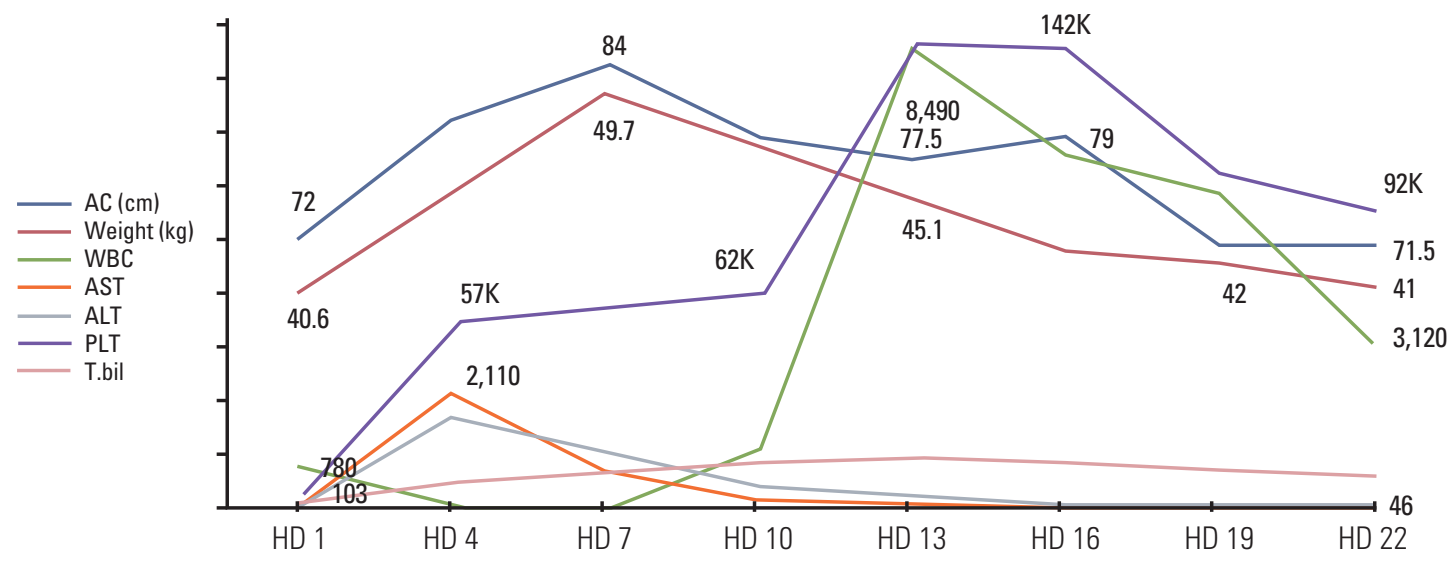

Defibrotide

Acetylcystein

AT-III

UDCA

Antibiotics

Mechanical ventilation

Fig. 2. Treatment and progress. AC, abdominal circumference; WBC, white blood cell; AST, aspartate transaminase; ALT, alanine transaminase; PLT, platelet; T.bil, total bilirubin; HD, hospital day; UDCA, ursodeoxycholic acid. 
vasopressor infusion, paracentesis of the ascites, glycerin enema, rifaximin, and lactulose (through an L-tube) and mechanical ventilation was started 4 days after the diagnosis of SOS. From the fourth day of defibrotide treatment, her condition gradually improved along with her recovery from myelosuppression. Defibrotide treatment was continued for 10 days. Her body weight began to decrease on $\mathrm{HD} 8$ and her fever gradually subsided and laboratory results showed improvement (Table 1, Fig. 2). She was weaned from a mechanical ventilator on HD 17. Abdominal US performed on HD 22 showed normalized blood flow of the right portal vein (Fig. 1B). She was discharged on HD 31, and her bilirubin level was normalized 2 months later. Her parents refused further chemotherapy, which was initially scheduled for 37 weeks. Subsequent surveillance imaging studies showed no evidence of recurrence of cancer or any sequelae to the liver for the following 11 months.

\section{Discussion}

Few cases of SOS that were not in the HSCT setting, in patients with Wilms tumor, acute lymphoblastic leukemia and medulloblastoma have been reported [4,5]. Some conventional chemotherapeutic agents are associated with the development of SOS, including actinomycin-D and cyclophosphamide. The doses of chemotoxic agents, their schedules, and the age of the patient are directly related to the risk of SOS. Actinomycin-D is used in the treatment of Wilms tumor and rhabdomyosarcoma, usually in combination with vincristine, anthracyclines, or alkylating agents [4]. Wilms tumor located at the right kidney, use of halothane as an anesthetic, and pre-existing liver diseases such as viral hepatitis have been suggested as risk factors for development of SOS $[4,6]$. The use of 6-tioguanine is an important risk factor for SOS in acute lymphoblastic leukemia [7]. A fatal case of SOS was reported in a medulloblastoma patient after receiving a single course of chemotherapy comprised of vincristine, carboplatin, and lomustine [4].

The reported incidence of SOS in rhabdomyosarcoma patients is $1.2 \%-5.3 \%$ [8,9]. The identified risk factors in this population include VAC chemotherapy and younger age ( $<36$ months) [9]. Strong association of an escalated dose of cyclophosphamide with the development of SOS was suggested [10]. While there has been no report of SOS in patients treated with IRS-I, -II, and -III chemotherapy protocols, SOS in patients treated with the IRS-IV protocol consisting of an escalated dose of cyclophosphamide has been reported $[2,8,10]$. SOS in patients treated with the above mentioned protocols after VAC with a cyclophosphamide dose of
$2.2 \mathrm{~g} / \mathrm{m}^{2}$ for a single dose administered within 30 minutes has been reported [10]. Although the total dose of cyclophosphamide administered to our patient was higher (3.6 $\mathrm{g} / \mathrm{m}^{2} /$ course $)$ than the reported dose $\left(2.2 \mathrm{~g} / \mathrm{m}^{2} /\right.$ course $)$ that allegedly caused SOS, prolonging the infusion time of cyclophosphamide might reduce the frequency of SOS [8]. Our patient received a daily dose of cyclophosphamide (1.2 $\mathrm{g} / \mathrm{m}^{2}$ ) for over 60 minutes, and there were no specific predisposing factors related to the development of SOS. While the exact cause of SOS in this setting remains unknown, we presume that both actinomycin-D and cyclophosphamide might be associated with the development of severe SOS in this case.

Severe $\mathrm{SOS}$ requires aggressive treatment and defibrotide is becoming one of the most promising agents. Defibrotide is a polydisperse oligonucleotide derived from porcine and bovine mucosa [11]. The following actions of defibrotide can help resolve the symptoms of SOS: (1) increasing the release of nitric oxide, prostacyclin, and prostaglandin E2; (2) decreasing the release of plasminogen activator inhibitor 1 ; and (3) decreasing the thrombin generation, tissue factor expression, and endothelin activity [12]. The therapeutic potential of defibrotide has largely been studied in patients who developed severe SOS after HSCT [4]. Regarding patients treated with conventional chemotherapy, successful treatment with defibrotide for SOS in childhood medulloblastoma, and adult patients with acute leukemia has been reported $[4,13]$. Cases of alveolar and gastrointestinal hemorrhage during defibrotide treatment, related to its fibrinolytic and anti-thrombotic property have been reported [14]. However, Cesaro et al. [5] suggested that defibrotide can be used safely in pediatric patients with close monitoring of hepatic flow with Doppler US, only in patients with portal hypertension in order to avoid hemorrhagic risk.

Albeit with a low incidence, patients receiving chemotherapy including actinomycin-D or cyclophosphamide (even in moderate doses) are at risk of developing SOS. SOS should be on the list of differential diagnoses for patients presenting with tender hepatomegaly, hyperbilirubinemia, and weight gain. For severe SOS, as in our case, meticulous care including defibrotide is required to save the patient's life and prevent sequelae to the liver.

\section{Conflicts of Interest}

Conflict of interest relevant to this article was not reported. 


\section{References}

1. Carreras E, Diaz-Beya M, Rosinol L, Martinez C, Fernandez-Aviles F, Rovira M. The incidence of veno-occlusive disease following allogeneic hematopoietic stem cell transplantation has diminished and the outcome improved over the last decade. Biol Blood Marrow Transplant. 2011;17: 1713-20.

2. Coppell JA, Richardson PG, Soiffer R, Martin PL, Kernan NA, Chen A, et al. Hepatic veno-occlusive disease following stem cell transplantation: incidence, clinical course, and outcome. Biol Blood Marrow Transplant. 2010;16:157-68.

3. Richardson PG, Ho VT, Cutler C, Glotzbecker B, Antin JH, Soiffer R. Hepatic veno-occlusive disease after hematopoietic stem cell transplantation: novel insights to pathogenesis, current status of treatment, and future directions. Biol Blood Marrow Transplant. 2013;19(1 Suppl):S88-90.

4. Kotecha RS, Buckland A, Phillips MB, Cole CH, Gottardo NG. Hepatic sinusoidal obstruction syndrome during chemotherapy for childhood medulloblastoma: report of a case and review of the literature. J Pediatr Hematol Oncol. 2014;36:76-80.

5. Cesaro S, Pillon M, Talenti E, Toffolutti T, Calore E, Tridello $\mathrm{G}$, et al. A prospective survey on incidence, risk factors and therapy of hepatic veno-occlusive disease in children after hematopoietic stem cell transplantation. Haematologica. 2005;90:1396-404.

6. Gentet JC, Bernard JL, Aimard L, Alfonsi SM, Raybaud C. Veno-occlusive disease in children after intensive chemoand radiotherapy and repeated halothane anesthesias: a report of 2 cases. Acta Oncol. 1988;27:579-81.

7. Wray L, Vujkovic M, McWilliams T, Cannon S, Devidas M, Stork L, et al. TPMT and MTHFR genotype is not associated with altered risk of thioguanine-related sinusoidal obstruction syndrome in pediatric acute lymphoblastic leukemia: a report from the Children's Oncology Group. Pediatr Blood Cancer. 2014;61:2086-8.

8. Ortega JA, Donaldson SS, Ivy SP, Pappo A, Maurer HM. Venoocclusive disease of the liver after chemotherapy with vincristine, actinomycin $\mathrm{D}$, and cyclophosphamide for the treatment of rhabdomyosarcoma. A report of the Intergroup Rhabdomyosarcoma Study Group. Childrens Cancer Group, the Pediatric Oncology Group, and the Pediatric Intergroup Statistical Center. Cancer. 1997;79:2435-9.

9. Arndt C, Hawkins D, Anderson JR, Breitfeld P, Womer R, Meyer W. Age is a risk factor for chemotherapy-induced hepatopathy with vincristine, dactinomycin, and cyclophosphamide. J Clin Oncol. 2004;22:1894-901.

10. Cecen E, Uysal KM, Ozguven A, Gunes D, Irken G, Olgun $\mathrm{N}$. Veno-occlusive disease in a child with rhabdomyosarcoma after conventional chemotherapy: report of a case and review of the literature. Pediatr Hematol Oncol. 2007;24:615-21.

11. Senzolo M, Germani G, Cholongitas E, Burra P, Burroughs AK. Veno occlusive disease: update on clinical management. World J Gastroenterol. 2007;13:3918-24.

12. Helmy A. Review article: updates in the pathogenesis and therapy of hepatic sinusoidal obstruction syndrome. Aliment Pharmacol Ther. 2006;23:11-25.

13. Bairey O, Kirgner I, Yakobi M, Hamdan A, Ben-Ari Z, Shaklai M. Clinical severe hepatic venoocclusive disease during induction treatment of acute monoblastic leukemia managed with defibrotide. Am J Hematol. 2002;69:281-4.

14. Bulley SR, Strahm B, Doyle J, Dupuis LL. Defibrotide for the treatment of hepatic veno-occlusive disease in children. Pediatr Blood Cancer. 2007;48:700-4. 